\title{
Studying Personalized Just-in-time Auditory Breathing Guides and Potential Safety Implications during Simulated Driving
}

\author{
Sebastian Zepf \\ MIT Media Lab \\ sebzepf@mit.edu
}

\author{
Asma Ghandeharioun \\ MIT Media Lab \\ asma_gh@mit.edu
}

\author{
Neska El Haouij \\ MIT Media Lab \\ neska@mit.edu \\ Javier Hernandez \\ MIT Media Lab \\ javierhr@mit.edu
}

\author{
Jinmo Lee \\ MIT Media Lab \\ jinmo@mit.edu
Rosalind W. Picard
MIT Media Lab
picard@mit.edu

\begin{abstract}
Driving can occupy a considerable part of our daily lives and is often associated with high levels of stress. Motivated by the effectiveness of controlled breathing, this work studies the potential use of breathing interventions while driving to help manage stress. In particular, we implemented and evaluated a closed-loop system that monitored the breathing rate of drivers in real-time and delivered either a conscious or an unconscious personalized acoustic breathing guide whenever needed. In a study with 24 participants, we observed that conscious interventions more effectively reduced the breathing rate but also increased the number of driving mistakes. We observed that prior driving experience as well as personality are significantly associated with the effect of the interventions, which highlights the importance of considering user profiles for in-car stress management interventions.
\end{abstract}

\section{CCS CONCEPTS}

- Human-centered computing $\rightarrow$ Auditory feedback; Empirical studies in $\mathrm{HCI}$; ・ Applied computing $\rightarrow$ Consumer health.

\section{KEYWORDS}

Breathing, Stress Management, Auditory Interventions, Closedloop Interventions, Automotive, Personalization

\section{ACM Reference Format:}

Sebastian Zepf, Neska El Haouij, Jinmo Lee, Asma Ghandeharioun, Javier Hernandez, and Rosalind W. Picard. 2020. Studying Personalized Just-in-time Auditory Breathing Guides and Potential Safety Implications during Simulated Driving. In Proceedings of the 28th ACM Conference on User Modeling, Adaptation and Personalization (UMAP '20), July 14-17, 2020, Genoa, Italy. ACM, New York, NY, USA, 9 pages. https://doi.org/10.1145/3340631.3394854

\section{INTRODUCTION}

Stress is very common in today's society, and affects a broad range of physical, psychological and behavioral conditions, including depression, anxiety, and sleep disorders $[8,9]$. Furthermore, stress has been shown to reduce job productivity and adversely affect

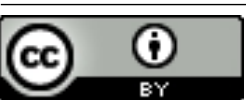

This work is licensed under a Creative Commons Attribution International 4.0 License. UMAP '20, July 14-17, 2020, Genoa, Italy

(C) 2020 Copyright held by the owner/author(s).

ACM ISBN 978-1-4503-6861-2/20/07.

https://doi.org/10.1145/3340631.3394854 overall well-being [10]. Driving, which can occupy a considerable part of daily life, is often associated with high levels of stress. In this context, certain levels of stress can be helpful to remain alert and attentive, but levels that are too high or too low can negatively impact driving performance and lead to life-threatening situations $[23,32,37,40]$.

While the stress response is usually associated with physiological indicators such as changes in core temperature, cardiovascular tone, and respiratory patterns, breathing has also been frequently used to counteract stress as it can be more easily controlled [7]. In particular, voluntary deep and slow breathing has been shown to induce physiological, affective, and cognitive calmness [14], and deep breathing has been shown to be effective in improving and maintaining cognitive processes such as concentration, attention, memory, and eye-hand coordination [36]. These benefits may be particularly helpful in the context of driving; however, it is an open question whether it is possible to use breathing to effectively manage stress without sacrificing driver attention, or how this may vary across individuals.

To address this question, we built and examined a closed-loop system in which one of two breathing interventions is triggered whenever the drivers' breathing rate exceeds a personalized threshold. We term one of the interventions "conscious" as it is designed to provide an acoustic guide for the driver to imitate with his or her breathing, and contrast the other one as "unconscious" as it slightly modulates background noise without demanding driver attention. (The name is not a proper full description; more detail is given below.) Both interventions were evaluated in a driving simulator study and compared against a neutral condition.

This work is organized as follows. First, we review prior work in the context of stress management interventions. Second, we describe our system design and experimental protocol using a car simulator. Third, we evaluate the two systems, examine the potential influence of user profile on the perception and effect of the interventions, and summarize insights from the user's qualitative feedback. Finally, we discuss the potential implications of our system for real-world driving and highlight areas of future work.

\section{RELATED WORK}

A myriad of research findings have identified breath modulation as an effective way of improving somatic, psychiatric, and psychological symptoms as well as overall well-being $[6,14,36]$. For example, Soni et al. [36] have shown that deep breathing is capable of enhancing and maintaining cognitive processes. The benefits include 
boosting concentration, attention, memory, eye-hand coordination, mental calculation ability, and learning. Additionally, Yogic breathing has proved to be an effective intervention to manage stress, anxiety, and depression [6]. This is complementary to the historical evidence finding Yogic breathing effective in enhancing well-being, mood, attention, mental focus, and stress tolerance [6]. Furthermore, slow breathing can lead to somatic benefits such as lowering blood pressure [14].

While breathing is a physiological process under autonomic control, the fact that it can be regulated consciously to achieve desired benefits makes it a unique signal to deliver health and wellness interventions [24]. However, trying to consciously manipulate breathing could have a toll on user's attention and be distracting, especially when the user is primarily engaged in another task [1]. Researchers have explored different ways of influencing breathing to balance the potential distraction with the added benefits of breathing interventions. These efforts have spread across a spectrum from requiring sustained attention to effortless interventions, even masking the true purpose of the study until after the experiment is completed to minimize the use of attentional resources.

On one end of the spectrum, we can find breathing interventions that require constant conscious attention. For instance, immersive virtual environments have been developed to promote sustained attention on breathing through generative soundtracks [31]. Other responsive environments have tried to reflect physiological signals in a dynamic architecture to promote awareness that may lead to slower breathing [33]. In another study, slow vibrations through an engineered sleeve with a pattern similar to calm breathing were generated and found to be capable of positively influencing perceived calmness [27]. To promote a more effortless approach, other researchers have investigated visual and audio oscillating stimuli with the aim to slow breathing of computer users engaged in a reading task. In that placebo-controlled study, researchers found that lowering breathing rate with barely perceptible rhythmic stimuli helped improve subjective calmness and focus without negatively impacting task performance [13]. On the other end of the spectrum, we can find breathing modulations that require no conscious attention. For instance, researchers have explored the possibility of cultivating calmness arising from stimuli that are individualized to the person's breathing during a sham task. In the corresponding study, music was composed in real-time according to the user's breathing patterns while they were focused on a reaction-time task, without knowledge about how the music was being generated or its potential purpose. Multiple physiological arousal indicators (breathing rate, electrodermal activity (EDA), heart rate, and slow cortical potentials measured in electrocardiographic signals) consistently showed a shift towards a calmer state [22].

The automotive context provides unique opportunities for measuring stress as well as designing calming interventions. Since drivers are sitting with limited motion, the measurement of physiological cues of stress such as EDA, heart rate, and respiration becomes easier. Additionally, the driver is in a confined space surrounded by a wide variety of intervention opportunities (e.g., dashboards, music, interior illumination). For instance, Hernandez et al. [16] suggested several examples of just-in-place stress interventions inside the car, such as recommending relevant music based on the emotional state, changing the tone of voice in the navigation system to be more empathic, or adjusting the behavior of the dashboard based on the user's perceived stress level. Other researchers have evaluated the potential use of calming interventions in simulated driving scenarios. For example, Paredes et al. [28] examined different types of haptic breathing patterns and showed a strong preference towards familiar movements which resembled breathing. In another study, Paredes et al. [29] compared haptic guidance based on vibrations and voice guidance based on spoken audio commands to promote slow breathing in a car simulator, and showed that both approaches can reduce breathing without affecting safety. Further studies have considered the automotive context to investigate the timing of the interventions [2] as well as how to promote attentiveness $[3,20]$.

\section{PROTOTYPE}

To investigate the effect of auditory feedback while driving, we designed a closed-loop system that is capable of providing two different types of audio interventions when a certain personalized threshold of breathing rate is exceeded.

\subsection{Audio Design}

As a basis for our system, we use white noise that is continuously played at a constant volume. In case of activation, the conscious intervention is played with higher volume on top of the basic noise, while the unconscious intervention modulates the volume of the noise without exceeding the constant level. While the participants are purposely introduced to the conscious guide, no instructions are given for the unconscious guide. For a better understanding, Figure 1 illustrates one cycle of each condition's audio shape.

3.1.1 Conscious Intervention. The goal of this intervention is to consciously guide the user's breathing rate through an easily perceptible auditory signal. In particular, the system uses two different harmonic chords to guide through the inhalation and exhalation of the breathing pattern. The volume of an F-major harmonic chord modulates with the shape of a Gaussian function in the inhalation period, and a C-major harmonic chord follows in the exhalation period. To further promote relaxation, the ratio of inhalation and exhalation period is set to 0.5 in one cycle of the intervention [39]. This intervention was motivated by the studies of Paredes et al. [28, 29] in which they effectively influenced drivers' breathing patterns with a "coach-like" voice guidance. In contrast to this work, we tried to minimize distraction by leveraging harmonic sounds instead of a voice guidance system. To introduce users to this conscious intervention, an explanatory voice sample uttering the following text was used: "The present auditory intervention is invented to regulate the breathing pattern of the study participants. The intervention consists of two different harmonic courts, F-Chord for inhalation [audio example], and C-Chord for exhalation [audio example]. Please listen to the intervention sound and follow three cycles with your breathe [audio example] - Great job. The intervention sound is not always on. When it is on, please try to regulate your breathing by following the sound. The provided breath rate of the sound can be different from this example depending on the context of the study. In any cases, your safety driving is the most important task rather than following the intervention. Hope you enjoy the participation in the study." 


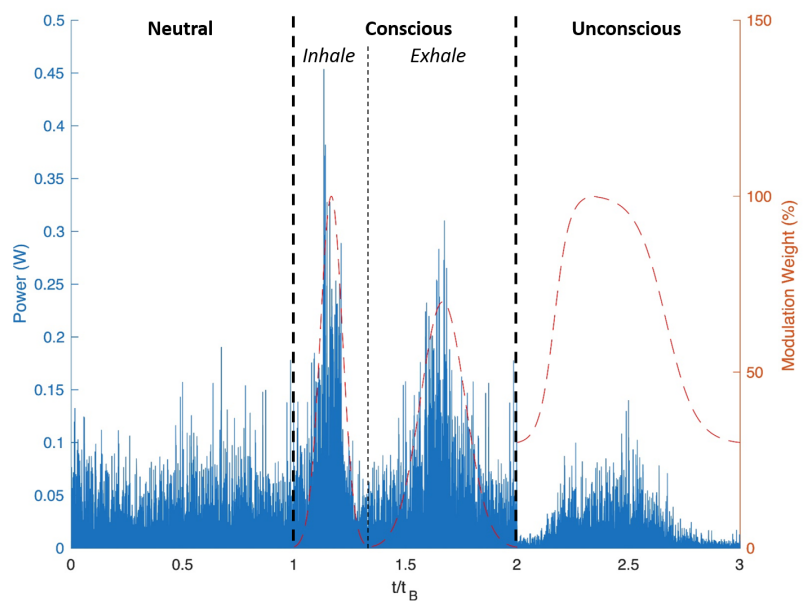

Figure 1: Audio shape and degree of modulation for one cycle of each condition.

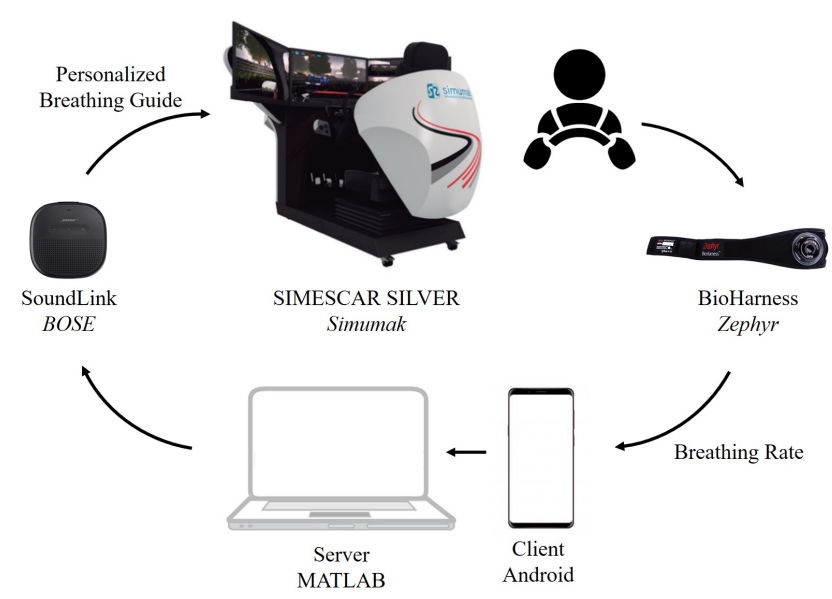

Figure 2: Schematic overview of the closed-loop system to provide personalized breathing regulation while driving.

3.1.2 Unconscious Intervention. The goal of this intervention is to unconsciously support calmness and focus via subtle volume changes of the background white noise. By the activation of the intervention, the volume of the background noise is periodically modulated between $30 \%$ and $100 \%$ of its volume with a Sigmoid function. The maximum volume and the modulation were designed to be barely noticeable during a typical driving task. This intervention was motivated by the study of Ghandeharioun and Picard [13] in which they successfully influenced breathing patterns with unconscious modulations in a less cognitive demanding office setting.

\subsection{System Implementation}

To provide just-in-time breathing interventions, we designed and implemented the closed-loop system shown in Figure 2.
To monitor the breathing of drivers, we used the chest-worn Zepyhr Bioharness which captures breathing and heart rate $(1 \mathrm{~Hz})$. The sensor was connected to a custom Android app that received live-streamed data from several sensors and transferred them to a MATLAB server through Bluetooth Low Energy (BLE). The server was responsible for the activation and deactivation of the interventions which were delivered through a wireless Bose SoundLink Micro Bluetooth speaker.

The breathing rate of the auditory interventions was personalized for each participant based on their resting physiology. Therefore, the system required a short calibration to collect the user's average mean breathing rate (MBR) at rest. Similar to [13], we set the personalized goal breathing rate $(\mathrm{GBR})$ to $120 \% \times \mathrm{MBR}$ with bottom and upper bounds of 5 and 15 breaths per minute, respectively. While driving, the system continuously compared the current breathing rate (CBR) and the personalized GBR, and used the information to activate the intervention whenever CBR was higher than GBR and deactivate it whenever CBR was below or equal to GBR. To ensure a smooth deactivation, the system ensured that a full breathing cycle was played without interruption.

\section{USER STUDY}

To evaluate the effect of both acoustic interventions, we conducted a controlled study in a simulator environment. The technical setup of the simulator as well as the study procedure is explained below.

\subsection{Simulation Environment}

We conducted a user study in the single-driver SIMESCAR SILVER driving simulator (SIMUMAK) shown in Figure 2. The simulator contains a steering wheel, three basic pedals (gas, brake, and clutch) as well as a gear lever (5 gears + reverse). While the simulator allows to switch settings between manual and automatic transmission, we used automatic transmission to help reduce cognitive load. Hardware switches for basic car features such as front light, indicators, horn, and windshield wipers as well as safety belt are also included. The simulated environment is provided by three 32 " monitors with a viewing angle of 135 degrees. In addition, a 10 " control panel is situated behind the steering wheel showing the dashboard information. The driver's seat is located on top of a 2-degree of freedom moving platform that allows an inclination of +-10 degrees to simulate longitudinal and lateral accelerations when breaking, accelerating, and turning. Vehicle sounds are provided with 5.1 surround speakers integrated into the headboard of the seat as well as the displays. During the driving sessions, the simulator captures telemetry information (e.g., speed, acceleration) as well as driving mistakes (i.e., infractions and crashes). To better understand the driver, a webcam was installed on top of the center screen to capture a frontal view of the drivers' face and upper body. In addition to the previously mentioned Zephyr sensor, participants were equipped with a Q-sensor biosensor manufactured by Affectiva which monitored EDA and skin temperature $(8 \mathrm{~Hz})$ to get more insights into the user's physiology. We extended the electrodes of the Q-sensor using copper cables to allow an attachment at the participant's left middle and index fingers since the fingers have a higher concentration of eccrine sweat glands. The Q-sensor itself was fixed at the participants' left wrist with an elastic wrist-worn 


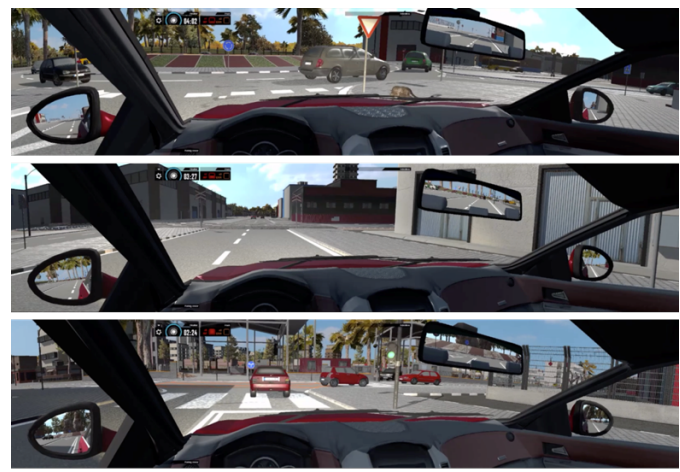

Figure 3: Screenshots of the driving environment showing other road users, street signage, and different types of roads.

band. It was always worn on the left so that it would encounter the same motion demands, e.g., activating the turn signal ${ }^{1}$.

From the different types of maps offered by the simulator, we selected city driving as it has been shown to be more stressful than highway driving [15] and to generate stronger changes in the stressrelated physiological signals [35]. Furthermore, we selected sunny weather during daytime with a medium amount of traffic to help simulate a typical day of city driving. Figure 3 shows representative driver's views that included roundabouts, crossings, street signage, and weather conditions.

\subsection{Protocol}

Figure 5 shows an overview of the experimental protocol which was pre-approved by the Institutional Review Board.

At the beginning of the study, participants were requested to complete the Big-Five Inventory (BFI) $[17,18]$ and the Patient Health Questionnaire (PHQ) [21]. These surveys were selected to capture relevant personal and health-related information that may have influenced driving performance as well as the potential effect of the interventions. The participants were then equipped with the biosensors and brought to the simulator to adjust the seat position. To calibrate the system, i.e. measuring the MBR during relaxation, participants were asked to remain calm for two minutes while listening to constant background noise. To allow some adaptation to the new setting, only the second minute was used to compute the personalized mean breathing rate. Figure 4 shows the distribution of the collected mean resting breathing rates as a histogram.

After the short calibration, the functionality of the simulator was explained to the participants who were told to follow the traffic rules during the whole experiment. Participants were then asked to perform a 5-minute training drive on the selected map to help familiarize with the simulation. To promote more natural driving, participants were allowed to choose their own route on the map. Finally, participants were introduced to the conscious breathing guide by playing the explanatory voice sample. After clarifying remaining questions, the main study started. The main study was

${ }^{1}$ In the future, we would recommend to switch it to always be worn on the right, incurring fewer motion artifacts when driving an automatic transmission.

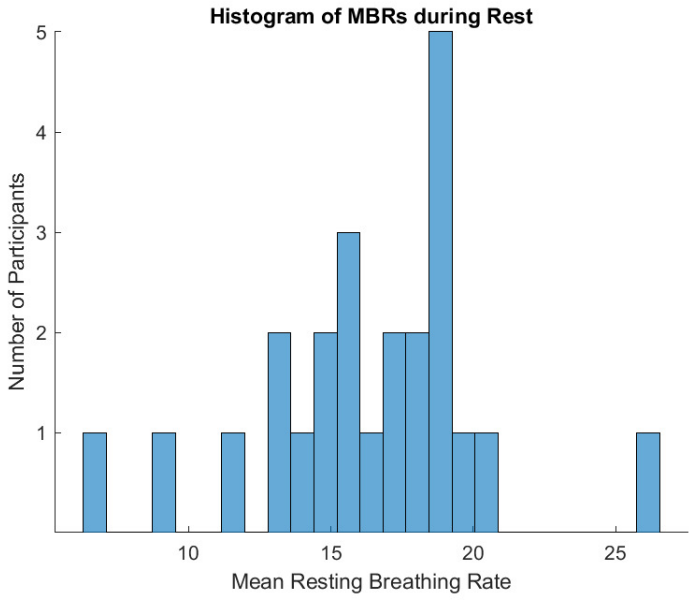

Figure 4: Histogram of the mean resting breathing rates (MBR) for the 24 participants.

designed as a within-subjects experiment and consisted of the following three 5-minute drives: (I) neutral (i.e., constant background noise), (II) conscious intervention (i.e., breathing guide + constant background noise), and (III) unconscious intervention (i.e., modulated background noise). To help minimize potential ordering effects, these conditions were performed in randomized order for each participant. After each of the drives, a short questionnaire on an Android tablet was handed to the participants to gather their subjective ratings in terms of valence, arousal, stress, and focus during the last driving segment. After completing the three conditions, participants were asked to report if they perceived any kind of acoustic feedback for each of them (reply options were "yes," "maybe" and "no"). If they answered "yes" or "maybe," they were additionally asked to report their subjective relaxation and distraction caused by the feedback. All of the following questions were requested on a 7-point Likert-scale:

- How pleasantly were you feeling during the last driving segment? (Valence)

- How energetic were you feeling during the last driving segment? (Arousal)

- How stressed were you feeling during the last driving segment? (Stress)

- How would you rate your focus on the driving task during the last driving segment? (Focus)

- How much did the audio feedback distract you from the driving task? (Distraction)

- How relaxing was the audio feedback for you? (Relaxation) The whole experiment lasted around 40 minutes and participants received $\$ 30$ as compensation.

\subsection{Data Description}

A total of 26 people completed the experiment. Participants were between 22 and 33 years old ( $M=26.7$ years, $S D=2.8$ years) except for one who was 67 years old. Among the participants, there were 14 females and 12 males and their prior real-life driving experience 


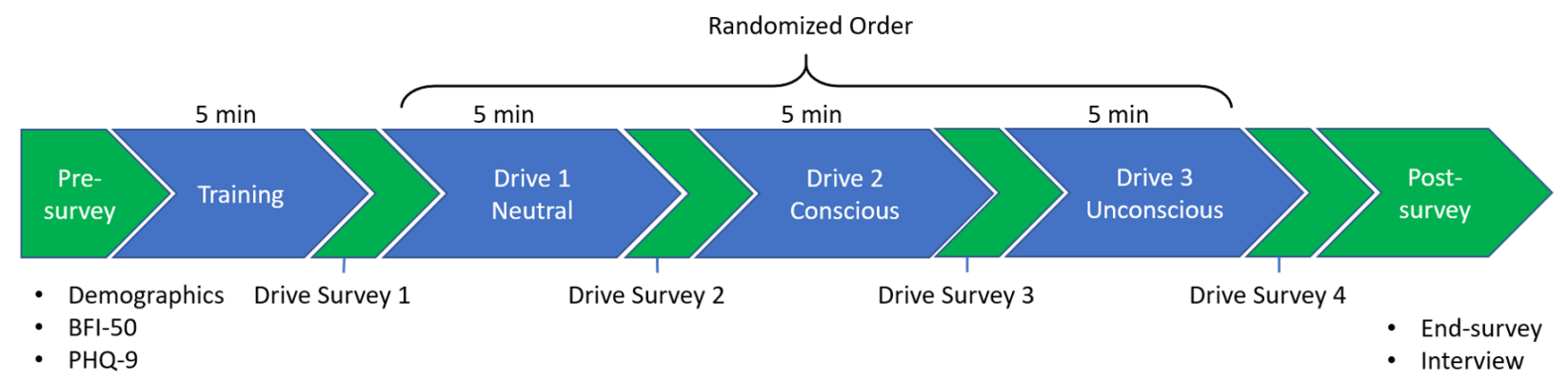

Figure 5: Overview of the experiment protocol.

ranged from one to eleven years $(M=6.4$ years, $S D=3.0$ years $)$, except for one who had around 50 years of driving experience. Eight of the participants had prior driving experience with the simulator. The data of two participants had to be excluded because their GBR was never exceeded and, consequently, no intervention was triggered. Thus, data from 24 participants was considered for the final analysis.

\section{RESULTS}

This section provides a quantitative and qualitative analysis of the collected data from the biosensors, the driving behavior, the subjective questionnaires, and the potential role of participants' profiles. All of the statistical analyses were performed using $\mathrm{R}$ (version 3.6.1). The significance level for the statistical tests was set at $5 \%$.

For the conscious intervention, the majority of participants (21 of 24) confirmed that they were able to perceive it. However, two of the participants stated that they were not able to listen to the breathing guide while driving and one participant answered "maybe" arguing that it was difficult to follow the intervention. For the unconscious intervention, 18 of the participants stated that they did not perceive it, while only four participants reported that they perceived the intervention. The remaining two participants answered "maybe." Although there was only constant background noise in the neutral condition, three participants believed that they perceived audio feedback, while another three participants answered "maybe." Overall, these reports support that the two interventions were, as originally intended, perceived and not perceived, respectively.

\subsection{Effects of the Intervention}

5.1.1 Driver Physiology. First, we analyzed the potential effect of the interventions on the mean breathing rate. To be able to compare the relative differences between the different conditions for all participants, a normalization using a division by the personalized goal breathing rate was conducted for each participant. For instance, if a participant had a GBR of 15 breaths per minute, the mean breathing rate for each condition was divided by 15 . In the following, the mean of the normalized breathing rate is denoted as MNBR. Figure 6 depicts the MNBR for all participants across conditions, showing that the values of the boxplot corresponding to the conscious are lower than for the neutral and unconscious conditions. Statistical analysis showed a main effect of the different interventions on the
MNBR (Friedman, $\chi^{2}=23.01, p<0.0005$ ). A posthoc comparison based on pairwise Wilcoxon test with Bonferroni correction revealed that the conscious condition significantly reduced MNBR in comparison with the unconscious condition $(p<0.0005)$ and the neutral setting $(p<0.0005)$. However, no difference of the MNBR in the unconscious condition was found compared to the neutral setting $(p>0.1)$. Furthermore, separating the participants who stated that they perceived acoustic feedback $(\mathrm{PU})(\mathrm{N}=6)$ from the participants who were not aware of the unconscious feedback at all (NPU) indicates that there is a trend towards a decreased MNBR for participants who believed they were receiving feedback (see Figure 7). In line with these results, the average time under GBR increased to $63 \%$ during the conscious condition in comparison to the unconscious (12\%) and the neutral condition (15\%). Overall, these findings suggest that the conscious intervention more successfully reduced the breathing rate.

For EDA, we extracted two features for each drive, namely the number of peaks and the mean tonic level [5]. For examination of both features, no significant differences were found across the conditions using Friedman test $(p=0.89$ for EDA peaks and $p=$ 0.44 for the mean tonic EDA). Based on previous work showing a correlation of EDA features with the number of present stressors while driving [15], these findings suggest that the driving task evoked similar levels of stress in each of the three drives, which supports comparability of the three conditions.

5.1.2 Driving Performance. To analyze driving performance, we considered two types of data provided by the simulation environment: number of crashes and infractions. To help amplify the relative differences across conditions, we normalized both the crashes and the infractions with the maximum values observed for each participant. As potential infractions we considered: exceeding the maximum allowed speed, moving too slow $(<50 \%$ of the allowed speed), and driving out of the lane. The maximum number of crashes observed for a single participant was seven, while ten participants performed the three conditions without any crashes. The highest numbers of crashes during a single drive was five. In terms of infractions, the maximum values for a single drive were twelve for exceeding the top speed, seven for moving too slow, and six for driving out of the lane.

Figure 8 shows the mean values of the normalized total number of crashes (MNC) for all participants across conditions as well as their respective standard errors. As it can be seen, there were more 


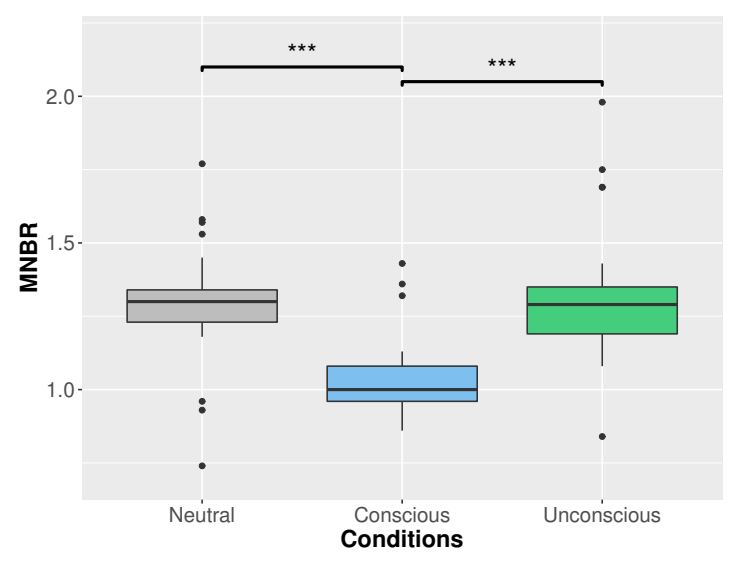

Figure 6: Boxplot showing the mean normalized breathing rate (MNBR) across conditions for all participants. Note that ${ }^{* * *}: p \leq 0.001$.

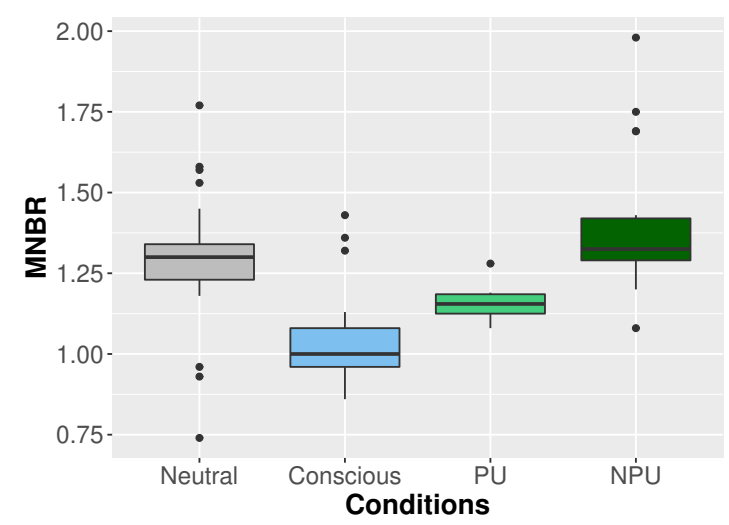

Figure 7: Boxplot showing the mean normalized breathing rate (MNBR) across conditions and whether the unconscious intervention was perceived (PU) or not (NPU) for all participants.

crashes on average during the conscious than during the neutral and unconscious conditions. When testing the difference of the mean of MNC between the three conditions, a significant difference was found (Friedman, $\chi^{2}=6, p=0.05$ ). In fact, the MNC was significantly higher in the conscious than in the neutral $(p=0.05)$ and unconscious $(p=0.03)$ condition based on Wilcoxon paired test with Bonferroni correction. This is evidence that following the conscious intervention may diminish attention towards the driving task. Furthermore, separating the participants who believed that they perceived acoustic feedback in the unconscious condition (PU) $(\mathrm{N}=6)$ revealed a tendency towards more crashes in comparison to the participants who did not perceive the intervention at all (NPU) (see Figure 9). This finding suggests that even perceiving the unconscious intervention demands some attentional resources that may affect the focus on the driving task.

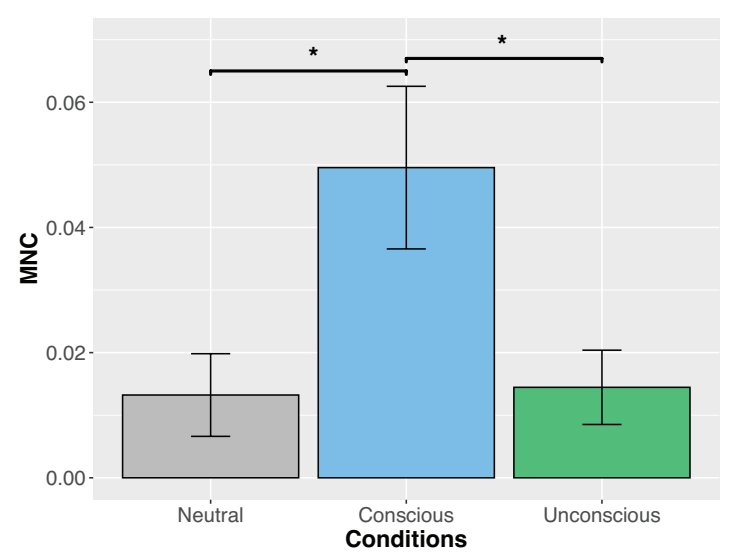

Figure 8: Mean normalized total number of crashes (MNC) and their standard error across conditions for all participants. Note that ${ }^{*}: p \leq 0.05$.

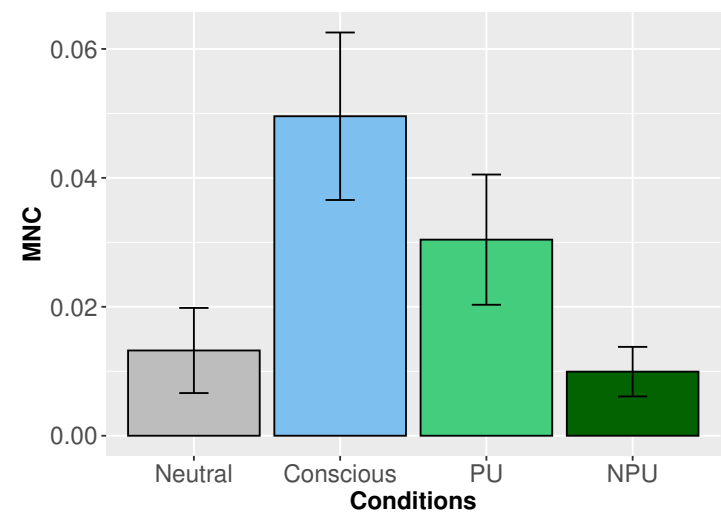

Figure 9: Mean normalized total number of crashes (MNC) and their standard error across conditions and whether the unconscious intervention was perceived (PU) or not (NPU) for all participants.

When comparing the overall infractions for the different settings, the conscious condition showed a tendency towards an increased number of normalized infractions in comparison to the other two conditions but no significant differences were found (Friedman, $\left.\chi^{2}=2.99, p=0.22\right)$. A more detailed analysis of the specific types of infractions showed a significant difference for top speed violations (Friedman, $\chi^{2}=9.08, p=0.01$ ). In fact, there were significantly more top speed violations in the conscious setting than in the neutral $(p=0.05)$ and unconscious $(p=0.02)$ conditions based on Wilcoxon paired test with Bonferroni correction. This indicates that there is less obeying of the speed limit in the conscious condition, which could be partially related to the increased cognitive load.

5.1.3 Subjective Self-Ratings. For the subjective self ratings of perceived valence, arousal, focus, and stress level, no significant differences were found based on a Friedman test $(p=0.41$ for valence, 


\begin{tabular}{crrr}
\hline Personality Trait & High & Medium & Low \\
\hline Extraversion & $\mathrm{N}=8,0.77(0.05)$ & $\mathrm{N}=7,0.56(0.03)$ & $\mathrm{N}=9,0.49(0.05)$ \\
Agreeableness & $\mathrm{N}=5,0.90(0.06)$ & $\mathrm{N}=12,0.79(0.04)$ & $\mathrm{N}=7,0.65(0.06)$ \\
Conscientousness & $\mathrm{N}=9,0.82(0.03)$ & $\mathrm{N}=7,0.71(0.04)$ & $\mathrm{N}=8,0.53(0.06)$ \\
Neuroticism & $\mathrm{N}=8,0.7(0.05)$ & $\mathrm{N}=9,0.49(0.04)$ & $\mathrm{N}=7,0.29(0.07)$ \\
Openness & $\mathrm{N}=7,0.87(0.04)$ & $\mathrm{N}=11,0.79(0.03)$ & $\mathrm{N}=6,0.63(0.08)$ \\
\hline
\end{tabular}

Table 1: Size (N), mean, and standard deviation (in brackets) for each considered group and personality trait.

$p=0.68$ for arousal, $p=0.64$ for focus, $p=0.08$ for stress). These findings are consistent with the fact that no significant difference was detected when analyzing the features extracted from the EDA.

\subsection{Role of the User Profile}

To examine the role of individual differences, we extended the analysis to consider the profile of each participant in terms of personality (captured by the BFI), depression (PHQ), driving and simulator experience. To categorize participants corresponding to the different co-variables, we computed the respective scores based on the participants' responses and clustered them into different groups. For the BFI dimensions, we assigned participants to three groups based on the LAH-method (Low-Average-High) [38]. Table 1 summarizes the sizes, the mean values and the standard deviations for the groups of each dimension of the BFI. In terms of the PHQ questionnaire, participants were classified into five groups ranging from no depression to severe depression using the depression severity according to [21]. In particular, ten participants were assigned to "none," four to "mild," seven to "moderate," and three to "moderately severe" depression level. No participant fell into the group of "severe." Due to the small number inside each group, we only considered two main groups: non-depressed (score ranging from 1 to 4,10 participants) and depressed (having a score of 10 or higher, 10 participants). In terms of the driving experience, participants were divided into two groups, once into less experienced (1-5 years of driving experience, 9 participants) and more experienced (more than 5 years, 15 participants) drivers. Finally, the study involved 7 drivers who previously had drove in a car simulator while 17 had not tried a simulator before.

Since the users' profile variables were not experimentally controlled, these data were not balanced per group. In this section, we report statistical differences in the physiology, driving performance, subjective ratings between the conscious and the unconscious intervention for each subgroup. All pairwise comparisons within the subgroups conducted in this section are based on the Wilcoxon paired test.

5.2.1 Driver Physiology. No significant differences were found for the effect of the interventions on the EDA features when considering depression level, previous simulator experience and personality (all $p>0.05$ ). For the driving experience, we found a significantly higher average tonic EDA level for participants with less driving experience during the conscious than in the unconscious condition $(p=0.05)$. This indicates a higher stress level for inexperienced drivers while following the conscious intervention.
5.2.2 Driving Performance. Several significant differences were found between the conscious and the unconscious conditions, when examining the driving performance considering the users' profiles. While the difference is not significant for the experienced drivers, the number of infractions is significantly higher in the conscious compared to the unconscious conditions for less experienced drivers $(p=0.04)$. This suggests that the factors related to driving experience are important for the design of in-car interventions that may require some conscious attention.

In terms of personality, we found that the infractions are significantly higher in the conscious than in the unconscious condition among the participants with high neuroticism $(p=0.05)$. A more detailed analysis of these infractions revealed that the major source of infractions was associated with the number of speed violations $(p=0.008)$. Further examination of the user profiles indicated a connection between the ratings for neuroticism and the depression level. The Spearman Correlation Coefficient confirmed a significant correlation between those two traits across participants ( $\rho=0.8, p<0.001)$, which is aligned with prior research $[19,26]$. These findings suggest an association between those characteristics and interventions demanding cognitive load, which should be carefully studied for real-life in-car applications.

5.2.3 Subjective Self-Ratings. Similar to the driving behavior, prior driving experience showed an association with the subjective selfratings. In particular, participants having less driving experience perceived the conscious condition as less pleasant and more stressful than the unconscious ( $p=0.05$ and $p=0.02$ respectively) condition.

Regarding the personality traits, the groups of low extraversion and medium openness showed significantly higher reported focus in the unconscious than in the conscious condition $(p=0.05$ for both). While no significant differences between the conscious and the unconscious conditions were found for perceived energy among the subgroups, the ratings for pleasure and stress were the most associated with the personality traits. In fact, the perceived pleasure and stress were significantly different (lower for the pleasure and higher for the stress) between the conscious and the unconscious conditions among the subgroups with medium agreeableness $(p=$ 0.05 and $p=0.03$ respectively), medium conscientousness ( $p=0.05$ and $p=0.02$ respectively), and high openness ( $p=0.05$ for stress). In addition, it is established that there is a relationship between the personality traits and the driving style and performance [4, 34]. These findings suggest that the personality traits are associated with the perception of the interventions and may be important to consider for the design of effective in-car breathing guides.

\section{QUALITATIVE USER FEEDBACK}

This section reviews qualitative responses of the participants after completion of the study which includes acceptability, preferences, and suggestions.

Several participants mentioned that the conscious intervention was a good fit for driving sections with low complexity, however, following the breathing guide in roads with high cognitive demands was challenging (7 participants). For instance, one participant stated "the conscious is easier for straight driving and difficult for complex situations." In line with this finding, two other participants stated 
that "the conscious intervention is distracting like having a phone call" and "[the breathing is] just another thing to do." One participant further clarified "the conscious [intervention] is especially adding stress during and after crashes." On the other hand, there were several opinions supporting the unconscious intervention may be more appropriate for real-world driving (4 participants).

Another relevant topic was the preference of listening to music instead of the breathing guide (4 participants). For instance, one participant said "breathing is good and helpful but [he] would prefer to listen to music." To solve this issue and to be able to speak with other passengers, one participant suggested to "put [the intervention] only on one ear somehow." In addition, one participant suggested that an "unconscious modulation of the music would be perfect."

Finally, there were several comments regarding the constant background noise. Some of the participants perceived the constant background noise as calming (3 participants). For instance, one participant stated that "he did not perceive the unconscious, but the constant noise was already calming." Additionally, another participant described the unconscious intervention as "positive distraction." However, there were also participants who commented that they ignored the background noise after some time (4 participants).

\section{DISCUSSION}

This paper studied two different approaches for personalized justin-time audio interventions to support calm breathing inside the car: one that acted as a conscious guide, and one that attempted to unconsciously influence breathing. Both approaches were implemented and evaluated in a driving simulator with 24 participants. Our findings show that the conscious audio guide better reduces the participants' breathing rate; however, it also significantly increased the number of crashes. In terms of the unconscious audio stimulation, we found a tendency towards a decrease in breathing rate when people reported to have perceived the intervention.

The post-study interviews revealed that the conscious guide was especially helpful in sections with low complexity but it was difficult to follow the breathing guide during more cognitive-demanding situations. These findings are further supported by the analysis of the user's profiles. In particular, previous experience played a crucial role in driving performance during the conscious intervention. These indicate that both focusing on the driving task and following the breathing guide at once may be too challenging for inexperienced drivers, which results in decreased driving performance and less effectiveness of the intervention. In terms of the unconscious intervention, the findings of Ghandeharioun \& Picard [13] are not aligned with our findings. In contrast to their study, where the noise modulation helped to support calmness and focus in an office setting, we could not find significant effects of the unconscious modulation on the breathing rate and subjective focus in our driving task. We believe this discrepancy may be partly associated with the higher complexity level of our task (i.e., city driving).

When considering user profiles, we found that personality is associated with the effect and the perception of the interventions. For instance, people with high neuroticism had significantly more infractions in the conscious and people with low extraversion felt significantly more focused in the unconscious condition. Correspondingly, future audio interventions may need to be tailored specifically to the user profile to maximize their positive and minimize their negative impact. For instance, users with different profiles may benefit from interventions with higher or lower volumes that help control the amount of attention that may be needed.

It is also important to note that this study suffered from some limitations. For instance, there are some major differences when comparing simulated and real-life driving, especially when considering potential threats and stress. Further, people usually have a high level of familiarity with their own vehicles which is difficult to replicate in a controlled laboratory study. We observed increased frequency of crashes than on real road, which may reflect that people needed time to adapt to the new environment. Finally, the duration of our drives was restricted to five minutes which may be too short to capture longer-term effects of breathing interventions. We believe that future studies should investigate the associations found in this paper with a larger number of participants, to verify if the breathing or the user profile is the basic underlying factor impairing driving performance. Also, future work may consider a real-world driving experiment for improved ecological validity.

The activation of the interventions in this study was based on the breathing rate, while several studies have shown progress in the measurement of higher level metrics such as stress [11, 15, 25]. We believe that a more sophisticated approach to measure stress can help enhance the timing of the activation and deactivation of the interventions, and thus increase both the effect of the intervention as well as benefit the experience of the users.

Finally, driver distraction can be categorized into four different types, namely visual, auditory, bio-mechanical, and cognitive [30], while previous research has shown that different types of distraction can have different effects of driving performance [12]. Accordingly, future approaches could explore multimodal signals (e.g. combining audio and haptic) which could allow to balance different distraction types to minimize performance impairment as well as to consider individual preferences and contextual needs of drivers (e.g., the driver is speaking on the phone or wants to listen to music).

\section{CONCLUSION}

This paper studied two approaches for an in-car audio intervention to support just-in-time calm breathing personalized to each driver's physiology: a more obvious "conscious" guide and another less obvious "unconscious" guide. While the conscious one significantly reduced the breathing rate, it also increased the number of driving mistakes. In addition, we found that aspects of a users' profile such as prior experience and personality may play critical roles when evaluating the effectiveness of such interventions and, therefore, more work is needed around this space. We are looking forward to a future when intelligent cars personalize the car environment to make the daily commute safer and less emotionally stressful.

\section{ACKNOWLEDGEMENTS}

This work is supported by the MIT Media Lab Consortium and the NTT Data Corporation which provided the car simulator as part of the Emotion Navigation Special Interest Group. We would also like to thank Craig Ferguson for his work on the data collection framework. 


\section{REFERENCES}

[1] Alexander T Adams, Jean Costa, Malte F Jung, and Tanzeem Choudhury. 2015. Mindless computing: designing technologies to subtly influence behavior. In Proceedings of the 2015 ACM International foint Conference on Pervasive and Ubiquitous Computing. ACM, 719-730.

[2] Stephanie Balters, Madeline Bernstein, and Pablo E Paredes. 2019. On-road stress analysis for in-car interventions during the commute. In Extended Abstracts of the 2019 CHI Conference on Human Factors in Computing Systems. ACM, LBW2110.

[3] Stephanie Balters, Elizabeth L Murnane, James A Landay, and Pablo E Paredes. 2018. Breath booster!: exploring in-car, fast-paced breathing interventions to enhance driver arousal state. In Proceedings of the 12th EAI International Conference on Pervasive Computing Technologies for Healthcare. ACM, 128-137.

[4] Orit [Taubman Ben-Ari] and Dalia Yehiel. 2012. Driving styles and their associations with personality and motivation. Accident Analysis \& Prevention 45 (2012) 416 - 422. https://doi.org/10.1016/j.aap.2011.08.007

[5] Wolfram Boucsein. 2012. Electrodermal activity. Springer Science \& Business Media.

[6] Richard P Brown and Patricia L Gerbarg. 2005. Sudarshan Kriya Yogic breathing in the treatment of stress, anxiety, and depression: part II - clinical applications and guidelines. Fournal of Alternative \& Complementary Medicine 11, 4 (2005), 711-717.

[7] Evangelia Charmandari, Constantine Tsigos, and George Chrousos. 2005. Endocrinology of the stress response. Annu. Rev. Physiol. 67 (2005), 259-284.

[8] Sheldon Cohen, Ronald C Kessler, and Lynn Underwood Gordon. 1997. Measuring stress: A guide for health and social scientists. Oxford University Press on Demand.

[9] Sheldon Cohen, David AJ Tyrrell, and Andrew P Smith. 1991. Psychological stress and susceptibility to the common cold. New England journal of medicine 325, 9 (1991), 606-612.

[10] Thomas W Colligan and Eileen M Higgins. 2006. Workplace stress: Etiology and consequences. Fournal of workplace behavioral health 21, 2 (2006), 89-97.

[11] Neska El Haouij, Jean-Michel Poggi, Raja Ghozi, Sylvie Sevestre-Ghalila, and Mériem Jaïdane. 2019. Random forest-based approach for physiological functional variable selection for driver's stress level classification. Statistical Methods \& Applications 28, 1 (01 Mar 2019), 157-185. https://doi.org/10.1007/s10260-0180423-5

[12] Johan Engström and Gustav Markkula. 2007. Effects of visual and cognitive distraction on lane change test performance. (2007).

[13] Asma Ghandeharioun and Rosalind Picard. 2017. BrightBeat: effortlessly influencing breathing for cultivating calmness and focus. In Proceedings of the 2017 CHI Conference Extended Abstracts on Human Factors in Computing Systems. ACM, 1624-1631.

[14] E Grossman, A Grossman, MH Schein, R Zimlichman, and B Gavish. 2001 Breathing-control lowers blood pressure. Fournal of human hypertension 15 , 4 (2001), 263.

[15] Jennifer Healey, Rosalind W Picard, et al. 2005. Detecting stress during realworld driving tasks using physiological sensors. IEEE Transactions on intelligent transportation systems 6, 2 (2005), 156-166.

[16] Javier Hernandez, Daniel McDuff, Xavier Benavides, Judith Amores, Pattie Maes and Rosalind Picard. 2014. AutoEmotive: bringing empathy to the driving experience to manage stress. In Proceedings of the 2014 companion publication on Designing interactive systems. ACM, 53-56.

[17] Oliver P John, Eileen M Donahue, and Robert L Kentle. 1991. The big five inventory-versions $4 \mathrm{a}$ and 54.

[18] Oliver P John, Sanjay Srivastava, et al. 1999. The Big Five trait taxonomy: History, measurement, and theoretical perspectives. Handbook of personality: Theory and research 2, 1999 (1999), 102-138.

[19] Pekka Jylhä and Erkki Isometsä. 2006. The relationship of neuroticism and extraversion to symptoms of anxiety and depression in the general population. Depression and anxiety 23, 5 (2006), 281-289.

[20] Nataliya Kosmyna, Caitlin Morris, Thanh Nguyen, Sebastian Zepf, Javier Hernandez, and Pattie Maes. (in press). AttentivU: Designing EEG and EOG Compatible Glasses for Physiological Sensing and Feedback in the Car. In Adjunct Proceedings of the 11th International Conference on Automotive User Interfaces and Interactive Vehicular Applications. ACM.
[21] Kurt Kroenke and Robert L Spitzer. 2002. The PHQ-9: a new depression diagnostic and severity measure. Psychiatric annals 32, 9 (2002), 509-515.

[22] Grace Leslie, Asma Ghandeharioun, Diane Y Zhou, and Rosalind W Picard. 2019. Engineering Music to Slow Breathing and Invite Relaxed Physiology. In 8th International Conference on Affective Computing and Intelligent Interaction, ACII 2019. Institute of Electrical and Electronics Engineers Inc.

[23] Gerald Matthews, Lisa Dorn, and A. Ian Glendon. 1991. Personality correlates of driver stress. Personality and Individual Differences 12, 6 (1991), 535 - 549. https://doi.org/10.1016/0191-8869(91)90248-A

[24] Neema Moraveji, Ben Olson, Truc Nguyen, Mahmoud Saadat, Yaser Khalighi, Roy Pea, and Jeffrey Heer. 2011. Peripheral paced respiration: influencing user physiology during information work. In Proceedings of the 24th annual ACM symposium on User interface software and technology. ACM, 423-428.

[25] Nermine Munla, Mohamad Khalil, Ahmad Shahin, and Azzam Mourad. 2015. Driver stress level detection using HRV analysis. In 2015 International Conference on Advances in Biomedical Engineering (ICABME). IEEE, 61-64.

[26] Peter Muris, Jeffrey Roelofs, Eric Rassin, Ingmar Franken, and Birgit Mayer. 2005. Mediating effects of rumination and worry on the links between neuroticism, anxiety and depression. Personality and Individual Differences 39, 6 (2005), 11051111.

[27] Athina Papadopoulou, Jaclyn Berry, Terry Knight, and Rosalind Picard. 2019. Affective Sleeve: Wearable Materials with Haptic Action for Promoting Calmness. In International Conference on Human-Computer Interaction. Springer, 304-319.

[28] Pablo Enrique Paredes, Nur Al-Huda Hamdan, Dav Clark, Carrie Cai, Wendy $\mathrm{Ju}$, and James A Landay. 2017. Evaluating in-car movements in the design of mindful commute interventions: exploratory study. fournal of medical Internet research 19, 12 (2017), e372.

[29] Pablo E Paredes, Yijun Zhou, Nur Al-Huda Hamdan, Stephanie Balters, Elizabeth Murnane, Wendy Ju, and James A Landay. 2018. Just breathe: In-car interventions for guided slow breathing. Proceedings of the ACM on Interactive, Mobile, Wearable and Ubiquitous Technologies 2, 1 (2018), 28.

[30] Michael Pettitt, Gary Burnett, and Alan Stevens. 2005. Defining driver distraction. Proc. 12th World Cong. on Intelligent Transport Systems (2005), 1-12.

[31] Mirjana Prpa, Kıvanç Tatar, Jules Françoise, Bernhard Riecke, Thecla Schiphorst, and Philippe Pasquier. 2018. Attending to breath: Exploring how the cues in a virtual environment guide the attention to breath and shape the quality of experience to support mindfulness. In Proceedings of the 2018 Designing Interactive Systems Conference. ACM, 71-84.

[32] AR Quimby and GR Watts. 1981. Human factors and driving performance. Technical Report.

[33] Holger Schnädelbach, Ainojie Irune, David Kirk, Kevin Glover, and Patrick Brundell. 2012. ExoBuilding: physiologically driven adaptive architecture. ACM Transactions on Computer-Human Interaction (TOCHI) 19, 4 (2012), 25.

[34] David C. Schwebel, Joan Severson, Karlene K. Ball, and Matthew Rizzo. 2006. Individual difference factors in risky driving: The roles of anger/hostility, conscientiousness, and sensation-seeking. Accident Analysis \& Prevention 38, 4 (2006), 801 - 810. https://doi.org/10.1016/j.aap.2006.02.004

[35] Ernst Simonson, Charles Baker, Neal Burns, Charles Keiper, OH Schmitt, and Stirling Stackhouse. 1968. Cardiovascular stress (electrocardiographic changes) produced by driving an automobile. American Heart fournal 75, 1 (1968), 125-135.

[36] Sunaina Soni, Lata N Joshi, and Anjum Datta. 2015. Effect of controlled deep breathing on psychomotor and higher mental functions in normal individuals. Indian 7 Physiol Pharmacol 59, 1 (2015), 41-47.

[37] Karl Halvor Teigen. 1994. Yerkes-Dodson: A law for all seasons. Theory \& Psychology 4, 4 (1994), 525-547.

[38] Edward Tighe and Charibeth Cheng. 2018. Modeling personality traits of filipino twitter users. In Proceedings of the Second Workshop on Computational Modeling of People's Opinions, Personality, and Emotions in Social Media. 112-122.

[39] Ilse Van Diest, Karen Verstappen, André E Aubert, Devy Widjaja, Debora Vansteenwegen, and Elke Vlemincx. 2014. Inhalation/exhalation ratio modulates the effect of slow breathing on heart rate variability and relaxation. Applied psychophysiology and biofeedback 39, 3-4 (2014), 171-180.

[40] Robert M Yerkes and John D Dodson. 1908. The relation of strength of stimulus to rapidity of habit-formation. Fournal of comparative neurology and psychology 18, 5 (1908), 459-482. 